\title{
Record Values from the Inverse Weibull Lifetime Model: Different Methods of Estimation
}

\author{
Khalaf S. Sultan \\ Department of Statistics and Operations Research, College of Science, King Saud University, Riyadh, Saudi Arabia \\ E-mail: ksultan@ksu.edu.sa \\ Received September 21, 2010; revised October 13, 2010; accepted November 15, 2010
}

\begin{abstract}
In this paper, we use the lower record values from the inverse Weibull distribution (IWD) to develop and discuss different methods of estimation in two different cases, 1) when the shape parameter is known and 2) when both of the shape and scale parameters are unknown. First, we derive the best linear unbiased estimate (BLUE) of the scale parameter of the IWD. To compare the different methods of estimation, we present the results of Sultan (2007) for calculating the best linear unbiased estimates (BLUEs) of the location and scale parameters of IWD. Second, we derive the maximum likelihood estimates (MLEs) of the location and scale parameters. Further, we discuss some properties of the MLEs of the location and scale parameters. To compare the different estimates we calculate the relative efficiency between the obtained estimates. Finally, we propose some numerical illustrations by using Monte Carlo simulations and apply the findings of the paper to some simulated data.
\end{abstract}

Keywords: Scale Parameter, Location Parameter, Best Linear Unbiased Estimates (BLUEs), Maximum Likelihood Estimates, Relative Efficiency and Monte Carlo Simulations

\section{Introduction}

Record values arise naturally in many real life applications involving data relating to weather, sport, economics and life testing studies. Many authors have studied record values and the associated statistics; see, for example, [1-7]. Reference [8] has established some recurrence relations for the moments of record values from the Gumbel distribution. Similar work has been carried out by $[9,10]$ for the generalized extreme value and exponential distributions, respectively. Reference [11] have also discussed some inferential methods based on record values from Gumbel distribution. [12,13] have discussed inferential techniques based on Weibull and generalized Pareto distributions, respectively. Reference [14] have compared different estimates based on record values from Weibull distribution. Reference [15] has considered different loss functions to develop the Bayesian estimates of the parameters of the IWD.

Let $X_{L(1)}, X_{L(2)}, \ldots, X_{L(n)}$ be the first $n$ lower record values from the IWD with density function $(p d f)$

$$
f(x)=c x^{-c-1} e^{-x^{-c}}, x \geq 0, c>0,
$$

and cumulative distribution function ( $c d f$ )

$$
F(x)=e^{-x^{-c}}, \quad x \geq 0, c>0 .
$$

The scale form of the IWD has its density function given by

$$
f(y)=\frac{c}{\sigma}\left(\frac{\sigma}{y}\right)^{c+1} \exp \left\{-\left(\frac{\sigma}{y}\right)^{c}\right\}, y \geq 0, \sigma>0
$$

while the location-scale IWD has its density function given by

$$
f(y)=\frac{c}{\sigma}\left(\frac{\sigma}{y-\theta}\right)^{c+1} \exp \left\{-\left(\frac{\sigma}{y-\theta}\right)^{c}\right\}, y \geq 0, \sigma>0, \theta \geq 0
$$

Reference [16] calls the IWD as the complementary Weibull distribution, while [17] call it the reciprocal Weibull distribution. Reference [18] have discussed some useful measures for the IWD.

The IWD plays an important role in many applications, including the dynamic components of diesel engines and several data set such as the times to breakdown of an insulating fluid subject to the action of a constant tension. [19] provide an interpretation of the IWD in the context of the load-strength relationship for a component. 
Reference [20] has fitted the IWD to the flood data. For more details on the IWD, see for example, [21].

The joint density function of the first $n$ lower record values $X_{L(1)}, X_{L(2)}, \ldots, X_{L(n)}$ is given by [5]

$$
f_{1,2, \ldots, n}\left(x_{L(1)}, x_{L(2)}, \ldots, x_{L(n)}\right)=f\left(x_{L(n)}\right) \prod_{i=1}^{n-1} \frac{f\left(x_{L(i)}\right)}{F\left(x_{L(i)}\right)} .
$$

From (1.5), the $p d f$. of $X_{L(m)}$ can be obtained as

$$
f_{m}(x)=\frac{1}{\Gamma(m)}\{-\log [F(x)]\}^{m-1} f(x), x \geq 0, m=1,2, \ldots,
$$

where $f($.$) and F($.$) are given in (1.1) and (1.2),$ respectively.

The the joint $p d f$ of $X_{L(m)}$ and $X_{L(n)}$ is given by

$$
\begin{aligned}
& f_{m, n}(x, y)=\frac{1}{\Gamma(m) \Gamma(n-m)}\{-\log [F(x)]\}^{m-1}\{-\log [F(y)] \\
& +\log [F(x)]\}^{n-m-1} \frac{f(x)}{F(x)} f(y), 0 \leq y<x<\infty \\
& \quad m, n=1,2, \ldots, m<n,
\end{aligned}
$$

where $f($.$) and F($.$) are given in (1.1) and (1.2),$ respectively.

The single and product moments of record values are (see [22])

$$
\mu_{m}^{(i)}=\frac{\Gamma\left(m-\frac{i}{c}\right)}{\Gamma(m)}, i<m c
$$

and

$$
\mu_{m, n}^{(i, j)}=\frac{\Gamma\left(m-\frac{i}{c}\right) \Gamma\left(n-\frac{i+j}{c}\right)}{\Gamma(m) \Gamma\left(n-\frac{i}{c}\right)}, i+j<n c, m<n,
$$

where $\Gamma(\cdot)$ is the gamma function.

In the following section, we derive the exact form of the BLUE scale parameter and present the BLUEs of the location-scale case of IWD. Next in Section 3, we derive the maximum likelihood estimates of the parameters of IWD. Finally, in Section 4 we discuss the relative efficiency of the obtained estimates.

\section{The BLUEs}

In this section, we derive the BLUE of the scale parameter and present the BLUEs of the location and scale parameters of the IWD.

\subsection{The Scale Case}

Let $Y_{L(1)}, Y_{L(2)}, \cdots, Y_{L(n)}$ denote the first $n$ lower record from the distribution in (1.3), and let $X_{L(i)}=Y_{L(i)} / \sigma, i=1,2, \ldots, n$ be the corresponding record values from the IWD in (1.1). Assume

$$
\begin{gathered}
Y=\left(Y_{L(1)}, Y_{L(2)}, \cdots, Y_{L(n)}\right)^{T}, \\
\mu=\left(\mu_{1}, \mu_{2}, \cdots, \mu_{n}\right)^{T} \\
1=\underbrace{(1,1, \cdots 1)^{T}}_{n}
\end{gathered}
$$

and $\sum=\left(\left(\sigma_{i, j}\right)\right), 1 \leq i, j \leq n$. Then, the BLUE of the scale parameter $\sigma$ is given by

$$
\sigma_{1}^{*}=\left\{\frac{\mu^{T} \Sigma^{-1}}{\mu^{T} \Sigma^{-1} \mu}\right\} Y=\sum_{i=1}^{n} a_{i} Y_{L(i)},
$$

and its variance is given by

$$
\operatorname{Var}\left(\sigma_{1}^{*}\right)=\sigma^{2}\left\{\frac{1}{\mu^{T} \Sigma^{-1} \mu}\right\},
$$

for details, refer to $[23,6]$. Since the double moment $\mu_{m, n}$ can be written as

$$
\begin{aligned}
& \mu_{m, n}=p_{m} q_{n}, p_{m}=\frac{\Gamma(m+1 / c)}{\Gamma(m)} \text { and } \\
& q_{n}=\frac{\Gamma(n-2 / c)}{\Gamma(n-1 / c)}-\frac{\Gamma(n-1 / c)}{\Gamma(n)}, n>2 / c,
\end{aligned}
$$

then the covariance matrix $\Sigma$ can be inverted analytically. Then $(i, j)^{\text {th }}$ element $\sigma^{i j}$ of $\Sigma^{-1}$ can be derived as

$$
\begin{aligned}
& \sigma^{i j}= \\
& \begin{cases}-\frac{c(i c-1) \Gamma(i+1)}{\Gamma(i-2 / c)}, & j=i+1, i=1,2, \ldots, n-1, \\
\frac{\left(2 i^{2} c^{2}-2 i c^{2}-4 i c+c^{2}+2 c+1\right) \Gamma(i)}{\Gamma(i-2 / c)}, & i=j=2 \text { and } n-1, \\
\frac{(c-1)^{2}}{\Gamma(1-2 / c)} & i=j=1, \\
\frac{q_{n-1}}{q_{n}} \frac{c(n c-c-1) \Gamma(n)}{\Gamma(n-1-2 / c)}, & i=j=n \\
0, & j>i+1 .\end{cases}
\end{aligned}
$$

From (1.8), (2.1) and (2.4), we have the BLUE of the scale parameter $\sigma$ as

$$
\sigma_{1}^{*}=a_{n} y_{L(n)}=\frac{\Gamma(n)}{\Gamma(n-1 / c)} Y_{L(n)},
$$

with variance is given by

$$
\operatorname{Var}\left(\sigma_{1}^{*}\right)=\left(\frac{\Gamma(n) \Gamma(n-2 / c)-\Gamma^{2}(n-1 / c)}{\Gamma^{2}(n-1 / c)}\right) \sigma^{2}, \mathrm{n}>2 / \mathrm{c} .
$$

It is clear from (2.5) that $\sigma^{*}$ is an unbiased estimate of 
$\sigma$.

Table 1 below shows the coefficients $a_{n}$ of the BLUE of $\sigma$ when $n=4$ to 7 and $c=3,4,5$.

The BLUE of $\sigma$ given in (2.5), can be used to construct $100(1-\alpha) \%$ confidence interval for $\sigma$ through the formula

$$
P\left(\frac{\Gamma(n-1 / c) \sigma_{1}^{*}}{\Gamma(n) T_{1-\alpha / 2}} \leq \sigma \leq \frac{\Gamma(n-1 / c) \sigma_{1}^{*}}{\Gamma(n) T_{\alpha / 2}}\right)=1-\alpha,
$$

where $T_{\alpha / 2}$ and $T_{1-\alpha / 2}$ are the lower and upper percentage points of the pivotal quantity

$$
T=\frac{\sigma_{1}^{*} \Gamma(n-1 / c)}{\sigma(n)}
$$

The cdf of $T$ is obtained to be $F_{T}(t)=\frac{\Gamma\left(n, t^{-c}\right)}{\Gamma(n)}$, where $\Gamma\left(n, t^{-c}\right)$ is the incomplete gamma function.

\section{Example}

Five lower record values are simulated from IWD with $c=3$ and $\sigma=1.0$ as follows: 3.07586, .90607, $68454, .62296, .62283$, by using the coefficients of the BLUE of the scale parameter given in Table 1, we have $\sigma_{1}^{*}=1.63139(.62283)=1.016079$, and the standard error of this estimate is S.E. $\left(\sigma_{1}^{*}\right)=1.016079 \sqrt{.02692}$ $=0.166711$. Now, we calculate the lower and upper $5 \%$ percentage points of of $T$ to be

$T_{0.025}=0.46048001250$ and $T_{0.975}=0.8508449965$. Then 95\% confidence interval for $\sigma$ can be calculated from (2.7) to be $(0.7320149148,1.352569185)$.

\subsection{The location-Scale Case}

Reference [15] has used the single and product moments of the record values from IWD. Then, he has used these moments to calculate the coefficients of BLUEs and the variances for records of size 4,5,6 and 7 and $c=3,4,5$ by using the forms (see [23]).

$$
\theta_{2}^{*}=\sum_{i=1}^{n} A_{i} Y_{L(i)}, \text { and } \sigma_{2}^{*}=\sum_{i=1}^{n} B_{i} Y_{L(i)}
$$

Table 2 represents the coefficients of the BLUEs $A_{i}$ and $B_{i}$ for records of sizes $4,5,6,7$ and the shape parameter $c=3,4,5$. while Table 3 represents the variances and covariances of the BLUEs in this case.

Reference [24] has used the coefficients of BLUEs in Table 2 to construct different confidence intervals for the location and scale parameters of IWD based on Edgeworth approximation and compare them with those based on Monte Calro simulation.

\section{The MLEs}

In this section, we discuss the maximum likelihood estimates of the parameters of IWD when the available data are lower record values. We consider two different cases: 1) the scale-parameter case and 2) the location-scale parameter case.

\subsection{The Scale-Parameter Case}

Let $Y_{L(1)}, Y_{L(2)}, \ldots, Y_{L(n)}$ represents the first $n$ lower record values from the scale-parameter IWD in (1.3), then the log likelihood function is given by

$$
\begin{aligned}
& \ell(c, \sigma)= \\
& n c \log (\sigma)+n \log (c)-\sigma^{c} y_{L(n)}^{-c}-(c+1) \sum_{i=1}^{n} \log y_{L(i)} .
\end{aligned}
$$

Now, we discuss two cases. They are:

1) When $\sigma$ unknown and $c$ known: the maximum likelihood estimate of $\sigma$ can be obtained from (3.1) as

$$
\hat{\sigma}_{1}=n^{1 / c} y_{L(n)} \text {. }
$$

2) When both of $\sigma$ and $c$ are unknown: the maximum likelihood estimates of $\sigma$ and $c$ can be obtained from (3.2) by solving the following two equations as

$$
\hat{c}=n\left(\sum_{i=1}^{n} \log \left(\log \left(x_{L(i)}\right)\right)-n \log \left(x_{L(n)}\right)\right)^{-1},
$$

and

$$
\hat{\hat{\sigma}}_{1}=n^{1 / \hat{c}} y_{L(n)} .
$$

From (3.2), we see that

Table 1. The coefficients of the BLUE of the scale parameter and the variance when $\sigma=1$.

\begin{tabular}{ccccccc}
\hline \multicolumn{2}{c}{$c=3$} & \multicolumn{2}{c}{$c=4$} & \multicolumn{2}{c}{$c=5$} \\
\hline$n$ & $a_{n}$ & $\operatorname{Var}\left(\sigma_{1}^{*}\right)$ & $a_{n}$ & $\operatorname{Var}\left(\sigma_{1}^{*}\right)$ & $a_{n}$ & $\operatorname{Var}\left(\sigma_{1}^{*}\right)$ \\
4 & 1.49544 & 03548 & 1.35655 & .01929 & 1.27818 & .01211 \\
5 & 1.63139 & .02692 & 1.44699 & .01476 & 1.34545 & .00931 \\
6 & 1.74792 & .02169 & 1.52314 & .01194 & 1.40151 & .00756 \\
7 & 1.85073 & .01815 & 1.58937 & .01003 & 1.44984 & .00636 \\
\hline
\end{tabular}


Table 2. The Coefficients of the BLUEs.

\begin{tabular}{ccccccc}
\hline & \multicolumn{2}{c}{$c=3$} & & \multicolumn{3}{c}{$c=5$} \\
\hline 4 & $A_{i}$ & $B_{i}$ & $A_{i}$ & $B_{i}$ & $A_{i}$ & $B_{i}$ \\
& -0.2353 & 0.3519 & -0.5294 & 0.7182 & -0.8421 & 1.0764 \\
& -1.4118 & 2.1112 & -1.7647 & 2.3939 & -2.1053 & 2.6909 \\
& -2.1176 & 3.1668 & -2.3529 & 3.1919 & -2.6316 & 3.3636 \\
& 4.7647 & -5.6299 & 5.6471 & -6.3040 & 6.5789 & -7.1309 \\
& -0.1400 & 0.2284 & -0.3383 & 0.4896 & -0.5591 & 0.7523 \\
& -0.8400 & 1.3704 & -1.1278 & 1.6319 & -1.3978 & 1.8807 \\
& -1.2600 & 2.0555 & -1.5038 & 2.1759 & -1.7473 & 2.3509 \\
& -1.6200 & 2.6428 & -1.8045 & 2.6111 & -2.0161 & 2.7126 \\
& 4.8600 & -6.2972 & 5.7744 & -6.9085 & 6.7204 & -7.6966 \\
& -0.0942 & 0.1647 & -0.2395 & 0.3649 & -0.4071 & 0.5706 \\
& -0.5653 & 0.9881 & -0.7985 & 1.2162 & -1.0178 & 1.4265 \\
& -0.8479 & 1.4821 & -1.0646 & 1.6216 & -1.2723 & 1.7831 \\
& -1.0902 & 1.9055 & -1.2776 & 1.9459 & -1.4680 & 2.0575 \\
& -1.3082 & 2.2866 & -1.4601 & 2.2239 & -1.6311 & 2.2861 \\
& 4.9058 & -6.8270 & 5.8403 & -7.3725 & 6.7964 & -8.1238 \\
& -0.0684 & 0.1266 & -0.1809 & 0.2875 & -0.3143 & 0.4556 \\
& -0.4104 & 0.7595 & -0.6029 & 0.9582 & -0.7857 & 1.1391 \\
& -0.6156 & 1.1393 & -0.8038 & 1.2776 & -0.9821 & 1.4239 \\
& -0.7915 & 1.4648 & -0.9646 & 1.5331 & -1.1332 & 1.6429 \\
& -0.9498 & 1.7578 & -1.1024 & 1.7521 & -1.2591 & 1.8255 \\
& -1.0959 & 2.0282 & -1.2249 & 1.9468 & -1.3686 & 1.9842 \\
& 4.9316 & -7.2763 & 5.8794 & -7.7552 & 6.8429 & -8.4712 \\
\hline
\end{tabular}

Table 3. The variances and covariances of the BLUEs when $\sigma=1$.

\begin{tabular}{ccccc}
\hline$c$ & $n$ & $\operatorname{Var}\left(\theta_{2}^{*}\right)$ & $\operatorname{Var}\left(\sigma_{2}^{*}\right)$ & $\operatorname{Cov}\left(\theta_{2}^{*}, \sigma_{2}^{*}\right)$ \\
\hline 3 & 4 & 0.3152 & 0.6834 & -0.4713 \\
3 & 5 & 0.1875 & 0.4901 & -0.3059 \\
3 & 6 & 0.1262 & 0.3803 & -0.2206 \\
3 & 7 & 0.0916 & 0.3103 & -0.1696 \\
4 & 4 & 0.3128 & 0.5646 & -0.4243 \\
4 & 5 & 0.1999 & 0.4138 & -0.2893 \\
4 & 6 & 0.1415 & 0.3255 & -0.2156 \\
4 & 7 & 0.1069 & 0.2680 & -0.1698 \\
5 & 4 & 0.3135 & 0.5055 & -0.4007 \\
5 & 5 & 0.2082 & 0.3739 & -0.2801 \\
5 & 6 & 0.1516 & 0.2960 & -0.2124 \\
5 & 7 & 0.1170 & 0.2447 & -0.1696 \\
\hline
\end{tabular}

$$
E\left(\hat{\sigma}_{1}\right)=n^{1 / c} E\left(\sigma x_{L(n)}\right),
$$

which upon using (1.8) gives

$$
E\left(\hat{\sigma}_{1}\right)=\sigma n^{1 / c} \frac{\Gamma(n-1 / c)}{\Gamma(n)} .
$$

This shows that

$$
\tilde{\sigma}_{1}=\frac{\Gamma(n)}{\Gamma(n-1 / c)} y_{L(n)},
$$

is an unbiased estimate for $\sigma$ in this case.

The variance of $\tilde{\sigma}_{1}$ is calculated to be

$$
\operatorname{Var}\left(\tilde{\sigma}_{1}\right)=\left(\frac{\Gamma(n) \Gamma(n-2 / c)-\Gamma^{2}(n-1 / c)}{\Gamma^{2}(n-1 / c)}\right) \sigma^{2}, \mathrm{n}>2 / \mathrm{c} .
$$

\section{Lemma 1}

The MLE of $\sigma$ given in (3.2) is asymptotically unbiased and its variance converges to zero as $n \rightarrow \infty$. 
Proof The proof can be easily done by using the expansion of gamma function see [24]

$$
\Gamma(z)=z^{z-1 / 2} \exp (-z) \sqrt{2 \pi}\left(1+\frac{1}{12 z}+\frac{1}{288 z^{2}}+O\left(\frac{1}{z^{3}}\right)\right) .
$$

\subsection{The Location-Scale Case}

When $Y_{L(1)}, Y_{L(2)}, \ldots, Y_{L(n)}$ represents the first $n$ lower record values from the location-scale parameter IWD given in (1.4), then the log likelihood function is given by

$$
\begin{aligned}
& l(c, \sigma, \theta)= \\
& n \log \left(\frac{c}{\sigma}\right)-\left(\frac{y_{L(n)}-\theta}{\sigma}\right)^{-c}-(c+1) \sum_{i=1}^{n}\left(\log \left(x_{L(i)}-\theta\right)-\log (\sigma)\right) .
\end{aligned}
$$

Now, we discuss two cases. They are:

1) When $c$ is known: the maximum likelihood estimates of $\theta$ and $\sigma$ can be obtained by solving the following two equations

$$
\begin{gathered}
\hat{\sigma}_{2}=n^{1 / c}\left(y_{L(n)}-\hat{\theta}_{2}\right), \\
(c+1) \sum_{i=1}^{n}\left(y_{L(i)}-\hat{\theta}_{2}\right)^{-1}-\frac{n c}{y_{L(n)}-\hat{\theta}_{2}}=0 .
\end{gathered}
$$

Table 4 below displays the bias and the estimated variance of the MLEs of the location and scale parameters in this case.

2) When $c$ is unknown: the maximum likelihood estimates of $\sigma, \theta$ and $c$ can be obtained from (3.9) by solving the following three equations as

$$
\begin{gathered}
\frac{n}{c}-\left(\frac{\sigma}{y_{L(n)}-\theta}\right)^{c} \log \left(\frac{\sigma}{y_{L(n)}-\theta}\right)-\sum_{i=1}^{n} \log \left(\frac{y_{L(i)}-\theta}{\sigma}\right)=0 \\
\sigma=n^{1 / c}\left(y_{L(n)}-\theta\right) \\
(c+1) \sum_{i=1}^{n}\left(y_{L(i)}-\theta\right)^{-1}-\frac{n c}{y_{L(n)}-\theta}=0
\end{gathered}
$$

\section{Relative Efficiency}

To compare between the BLUEs and the MLEs obtained in Sections 2 and 3, we calculate the relative efficiency in some cases as follows:

1) For the scale case we have $\operatorname{RE}\left(\sigma_{1}^{*}, \tilde{\sigma}_{1}\right)=1$,

2) For the location-scale case we have

$$
\operatorname{RE}\left(\theta_{2}^{*}, \hat{\theta}_{2}\right)=\frac{\operatorname{Var}\left(\theta_{2}^{*}\right)}{S_{\hat{\theta}_{2}}^{2}} \text {, and } \operatorname{RE}\left(\sigma_{2}^{*}, \hat{\sigma}_{2}\right)=\frac{\operatorname{Var}\left(\sigma_{2}^{*}\right)}{S_{\hat{\sigma}_{2}}^{2}} \text {. }
$$

Table 5 given below show the relative efficiency be-

Table 4. The bias and estimated variance of the MLEs.

\begin{tabular}{cccccc}
\hline$c$ & $n$ & $\operatorname{Bias}\left(\hat{\theta}_{2}\right)$ & $\operatorname{Bias}\left(\hat{\sigma}_{2}\right)$ & $S_{\hat{\theta}_{2}}^{2}$ & $S_{\hat{\sigma}_{2}}^{2}$ \\
\hline 3 & 4 & 0.05838 & 0.04222 & 0.35204 & 0.96583 \\
3 & 5 & 0.04556 & 0.01134 & 0.30296 & 0.96390 \\
3 & 6 & 0.06768 & -0.11985 & 0.21086 & 0.45111 \\
3 & 7 & 0.18492 & -0.41586 & 0.14638 & 0.32753 \\
4 & 4 & 0.17717 & -0.13855 & 0.39000 & 0.58523 \\
4 & 5 & 0.06416 & -0.00930 & 0.35768 & 0.48147 \\
4 & 6 & 0.10480 & -0.13350 & 0.21182 & 0.39013 \\
4 & 7 & 0.02483 & -0.01781 & 0.15638 & 0.34454 \\
5 & & & & \\
5 & 4 & 0.05524 & 0.01944 & 0.51265 & 0.92268 \\
5 & 5 & 0.15418 & -0.14972 & 0.26795 & 0.42049 \\
5 & 6 & 0.14928 & -0.16165 & 0.21410 & 0.38703 \\
\hline
\end{tabular}

Table 5 . The relative efficiency.

\begin{tabular}{ccccccc}
\hline \multicolumn{2}{c}{$c=3$} & \multicolumn{2}{c}{$c=4$} & \multicolumn{2}{c}{$c=5$} \\
\hline$n$ & $R E\left(\theta_{2}^{*}, \hat{\theta}_{2}\right)$ & $R E\left(\sigma_{2}^{*}, \hat{\sigma}_{2}\right)$ & $R E\left(\theta_{2}^{*}, \hat{\theta}_{2}\right)$ & $R E\left(\sigma_{2}^{*}, \hat{\sigma}_{2}\right)$ & $R E\left(\theta_{2}^{*}, \hat{\theta}_{2}\right)$ & $R E\left(\sigma_{2}^{*}, \hat{\sigma}_{2}\right)$ \\
4 & 0.89535 & 0.70758 & 0.80205 & 0.96475 & 0.61153 & 0.54786 \\
5 & 0.61889 & 0.50846 & 0.55888 & 0.85945 & 0.77701 & 0.88920 \\
6 & 0.59850 & 0.84303 & 0.66802 & 0.83434 & 0.70808 & 0.76480 \\
7 & 0.62577 & 0.94739 & 0.68359 & 0.77785 & 0.62721 & 0.86220 \\
\hline
\end{tabular}


-tween the different estimates of the location and scale parameters. From Table 5, we see that, the BLUEs give more efficiency than the MLEs.

\section{Acknowledgements}

The author would like to thank the referees for their helpful comments, which improved the presentation of the paper. Also, the author would like to thank the Research Center, College of Science, King Saud University, for funding the project (Stat/2008/31).

\section{References}

[1] K. N. Chandler, "The Distribution and Frequency of Record Values," Journal of the Royal Statistical Society Series $B$, Vol. 14, 1952, pp. 220-228.

[2] V. B. Nevzorov, "Records," Theory of Probability and Its Applications, Vol. 32, 1988, pp. 201-228.

[3] H. N. Nagaraja, "Record Values and Related Statistics-A Review," Communications in Statistics-Theory and Methods, Vol. 17, 1988, pp. 2223-2238.

[4] M. Ahsanullah, "Introduction to Record Values," Ginn Press, Needham Heights, Massachusetts, 1988.

[5] M. Ahsanullah, "Record values, The Exponential Distribution: Theory, Methods and Applications," In: N. Balakrishnan and A.P. Basu Eds., Gordon and Breach Publishers, Newark, New Jersey, 1995.

[6] B. C. Arnold, N. Balakrishnan and H. N. Nagaraja, “A First Course in Order Statistics,” John Wiley \& Sons, New York, 1992.

[7] B. C. Arnold, N. Balakrishnan and H. N. Nagaraja, "Records,” John Wiley \& Sons, New York, 1998.

[8] N. Balakrishnan, M. Ahsanullah and P. S. Chan, "Relations for Single and Product Moments of Record Values from Gumbel Distribution,” Statistics \& Probability Letters, Vol. 15, No. 3, 1992, pp. 223-227.

[9] N. Balakrishnan, P. S. Chan and M. Ahsanullah, "Recurrence Relations for Moments of Record Values from Generalized Extreme Value Distribution," Communications in Statistics-Theory and Methods, Vol. 22, No. 5, 1993, pp. 1471-1482.

[10] N. Balakrishnan and M. Ahsanullah, "Relations for Single and Product Moments of Record Values from Exponential Distribution," Journal of Applied Statistical Science, Vol. 2, 1995, pp. 73-87.

[11] N. Balakrishnan, M. Ahsanullah, and P. S. Chan, "On the Logistic Record Values and Associated Inference," Journal of Applied Statistics, Vol. 2, pp. 233-248.
[12] K. S. Sultan and N. Balakrishnan, "Higher Order Moments of Record Values from Rayleigh and Weibull Distributions and Edgeworth Approximate Inference," Journal of Applied Statistical Science, Vol. 9, 1999, pp. 193-209.

[13] K. S. Sultan and M. E. Moshref, "Higher Order Moments of Record Values from Generalized Pareto Distribution and Associated Inference,” Metrika, Vol. 51, 2000, pp. 105- 116.

[14] A. A. Soliman, A. H. Abd Ellah and K. S. Sultan, “Comparison of Estimates Using Record Statistics from Weibull Model: Bayesian and Non-Bayesian Approaches," Computational Statistics and Data Analysis, Vol. 51, No. 3, 2006, pp. 2065-2077.

[15] K. S. Sultan, "Higher Order Moments of Record Values from the Inverse Weibull Lifetime Model and Edgeworth Approximate Inference," International Journal of Reliability and Applications, Vol. 8, No. 1, 2007, pp. 1-16.

[16] A. Drapella, "Complementary Weibull Distribution: Unknown or Just Forgotten," Quality and Reliability Engineering International, Vol. 9, 1993, pp. 383-385.

[17] G. S. Mudholkar and G. D. Kollia, "Generalized Weibull family: A Structural Analysis," Communications in Statistics-Theory and Methods, Vol. 23, 1994, pp. 11491171.

[18] R. Jiag, D. N. P. Murthy and P. Ji, "Models Involving two Inverse Weibull Distributions," Reliability Engineering and System Safety, Vol. 73, No. 1, 2001, pp. 73-81.

[19] R. Calabria and G. Pulcini, "On the Maximum Likelihood and Least-Squares Estimation in the Inverse Weibull Distributions,” Statistica Applicata, Vol. 2, No. 1, 1990, pp. 53-66.

[20] M. Maswadah, “Conditional Confidence Interval Estimation for the Inverse Weibull Distribution Based on Censored Generalized Order Statistics,” Journal of Statistical Computation Simulation, Vol. 73, No. 12, 2003, pp. 887898.

[21] D. N. P. Murthy, M. Xie and R. Jiang, "Weibull Models," John Wiley \& Sons, New York, 2004.

[22] E. M. Nigm and R. F. Khalil, "Record Values from Inverse Weibull Distribution and Associated Inference," Bulletin of the Faculty of Science, 2006.

[23] N. Balakrishnan and A. C. Cohen, "Order Statistics and Inference: Estimation Methods,” Academic Press, San Diego, 1991.

[24] M. Abramowitz and I. A. Stegun, "Handbook of Mathematical Functions with Formulas, Graphs and Mathematical Tables,” Dover, New York, 1972. 